\title{
Implementing a Birth Dose of Hepatitis B Vaccine in Africa: Findings from Assessments in 5 Countries
}

\author{
Edna Moturi', Carole Tevi-Benissan ${ }^{1 *}$, José E. Hagan², Stephanie Shendale ${ }^{3}$, David Mayenga' ${ }^{1}$ Daniel Murokora', \\ Minal Patel ${ }^{2}$, Karen Hennessey ${ }^{3}$, Richard Mihigo ${ }^{1}$ \\ 'World Health Organization Regional Office for Africa, Brazzaville, Republic of Congo \\ ${ }^{2}$ Global Immunization Division, Centers for Disease Control and Prevention, Atlanta, GA, USA \\ ${ }^{3}$ World Health Organization, Expanded Programme on Immunization, Geneva, Switzerland
}

Article Info

\section{Article Notes}

Published: August 02, 2018

\section{*Correspondence:}

Dr. Carole Tevi-Benissan, World Health Organization

Regional Office for Africa, Brazzaville, Republic of Congo; Email: tevibenissanc@who.int

(c) 2018 Tevi-Benissan C. This article is distributed under the terms of the Creative Commons Attribution 4.0 International License.

\section{Keywords:}

Hepatitis

Birth Dose

Africa

WHO

\section{ABSTRACT}

Introduction: Few African countries have introduced a birth dose of hepatitis B vaccine (HepB-BD) despite a World Health Organization (WHO) recommendation. HepB-BD given within 24 hours of birth, followed by at least two subsequent doses, is $90 \%$ effective in preventing perinatal transmission of hepatitis $B$ virus. This article describes findings from assessments conducted to document the knowledge, attitudes, and practices surrounding HepB-BD implementation among healthcare workers in five African countries.

Methods: Between August 2015 and November 2016, a series of knowledge, attitude and practices assessments were conducted in a convenience sample of public and private health facilities in Botswana, the Gambia, Namibia, Nigeria, and São Tomé and Príncipe (STP). Data were collected from immunization and maternity staff through interviewer-administered questionnaires focusing on HepB-BD vaccination knowledge, practices and barriers, including those related to home births. HepB-BD coverage was calculated for each visited facility.

Results: A total of 78 health facilities were visited: STP 5 (6\%), Nigeria 23 (29\%), Gambia 9 (12\%), Botswana 16 (21\%), and Namibia 25 (32\%). Facilities in the Gambia attained high total coverage of $84 \%$ (range: $60-100 \%$ ) but low timely estimates $7 \%(16-28 \%)$ with the median days to receiving HepB-BD of 11 days (IQR: $6-16$ days). Nigeria had low total (23\% [range: $12-40 \%$ ]), and timely (13\% [range: $2-21 \%$ ]) HepB-BD estimates. Facilities in Botswana had high total (94\% [range: $80-100 \%])$, and timely (74\% [range: $57-88 \%$ ]) HepB$\mathrm{BD}$ coverage. Coverage rates were not calculated for STP because the maternal Hepatitis B virus (HBV) status was not recorded in the delivery registers. The study in Namibia did not include a coverage assessment component. Barriers to timely HepB-BD included absence of standard operating procedures delineating staff responsible for HepB-BD, not integrating HepB-BD into essential newborn packages, administering HepB-BD at the point of maternal discharge from facilities, lack of daily vaccination services, sub-optimal staff knowledge about HepB-BD contraindications and age-limits, lack of outreach programs to reach babies born outside facilities, and reporting tools that did not allow for recording the timeliness of HepB-BD doses.

Discussion: These assessments demonstrate how staff perceptions and lack of outreach programs to reach babies born outside health facilities with essential services are barriers for implementing timely delivery of HepB-BD vaccine. Addressing these challenges may accelerate $\mathrm{HepB}-\mathrm{BD}$ implementation in Africa.

\section{Introduction}

Hepatitis B virus (HBV) is a major cause of cirrhosis, liver cancer, and end-stage liver disease ${ }^{1,2}$. HBV is highly infectious and is 
transmitted by exposure to infected blood and other body fluids such as semen and vaginal fluid ${ }^{3}$. Most morbidity from HBV is due to chronic infection. An estimated 240 million people are infected with HBV worldwide, and more than 686,000 deaths are attributable to chronic HBV complications annually ${ }^{2,4,5}$. The likelihood of developing chronic HBV is highest if infection occurs at time of birth, and approximately $70-90 \%$ of persons infected perinatally progress to develop chronic HBV infection ${ }^{3,6,7}$.

With only $16 \%$ of the world's population, countries in the World Health Organization's (WHO) African Region are disproportionally affected, and have the highest endemicity worldwide (8.83\%, defined as having a chronic hepatitis B surface antigen (HBsAg) population prevalence of $\geq 8 \% \%^{8,9}$. Despite the availability of an effective vaccine, approximately $5-10 \%$ of the adult population is chronically infected and chronic HBV is a leading cause of death for young adults in the region, most of who are unaware of their infection until the disease has progressed to late stages ${ }^{2,10,11}$. In highly endemic settings, HBV is mainly transmitted perinatally from mother-to-child at the time of birth, and through horizontal transmission during early childhood ${ }^{12}$. WHO recommends universal vaccination with a monovalent dose of hepatitis B (HepB) vaccine (HepB-BD) given within 24 hours of birth, followed by at least two subsequent doses ${ }^{3}$. Vaccination has been shown to dramatically reduce the post-vaccination prevalence of HBsAg carriage in children less than 5 years $^{13}$. Timely HepB-BD (given within 24 hours after birth), followed by at least two doses is $90 \%$ effective at preventing perinatal transmission ${ }^{3,7}$.

All African countries have incorporated HepB vaccine in their Expanded Programme for Immunization (EPI) schedules. The vaccine is usually given as three doses of the pentavalent vaccine (diphtheria, tetanus, pertussis, Haemophilus influenza type B, and HepB) at 6, 10, and 14 weeks of age ${ }^{11}$. Regional coverage for the third dose of HepB (HepB3) has increased from 5\% in 2001 to $76 \%$ in 2015 ; however, this is below the global coverage of $84 \%{ }^{14}$. Individual country estimates range from $16 \%$ in Equatorial Guinea to $98 \%$ in Rwanda, Seychelles, Swaziland and the United Republic of Tanzania, with significant variation at district level. African countries have been slow to implement a 2009 WHO recommendation to introduce HepB-BD, and so far only $23 \%$ of children in the region are benefiting from the vaccine ${ }^{15}$. These children reside in 11 countries that have introduced HepB-BD (Algeria, Angola, Botswana, Cape Verde, the Gambia, Mauritania, Mauritius, Namibia, Nigeria, São Tomé and Príncipe [STP], and Senegal) ${ }^{14}$. In November 2014, during the Sixty-fourth session of the Regional Committee, the African Region adopted a resolution to reduce chronic $\mathrm{HBV}$ infection to less than $2 \%$ in children under 5 years of age, and to introduce HepB-BD in at least 25 countries by the end of $2020^{16}$.
Timely HepB-BD implementation poses programmatic challenges which should be considered prior to its introduction ${ }^{17,18}$. Even the Gambia which was one of the first countries to introduce HepB-BD, is reaching less than $3 \%$ of newborns with timely HepB-BD ${ }^{19,20}$. Multiple factors have been documented to affect implementation of HepB-BD ${ }^{21-23}$. Of these, human resource factors e.g., staff shortages, lack of training opportunities, poor attitude and gaps in knowledge among healthcare staff are frequently associated with poor uptake of immunization programs in Africa ${ }^{24,25}$. The effect of healthcare workers (HCWs) is especially important when it comes to implementing HepB$\mathrm{BD}$ given the timing recommendations of the vaccine.

With the recent resolution to increase timely HepB-BD coverage in the region, is important to first understand the experiences from the few countries that have already introduced HepB-BD. This will help guide other countries who are in the process of operationalizing HepB-BD or seeking to strengthen existing programmes. This paper presents findings from assessments conducted to determine the level of knowledge, attitudes, and practices surrounding HepB-BD administration among HCWs in five African countries.

\section{Methods}

Between August 2015 and November 2016, crosssectional studies were carried out in select administrative regions in Botswana, the Gambia, Namibia, Nigeria and STP (Figure 1). The regions were selected based on the previous years' reported HepB-BD coverage and identification as a priority area by EPI staff based on low immunization system performance as indicated by coverage with three doses of DTP (DTP3) vaccine. Within each region, at least two provinces were selected based on HepB-BD coverage and operational feasibility.
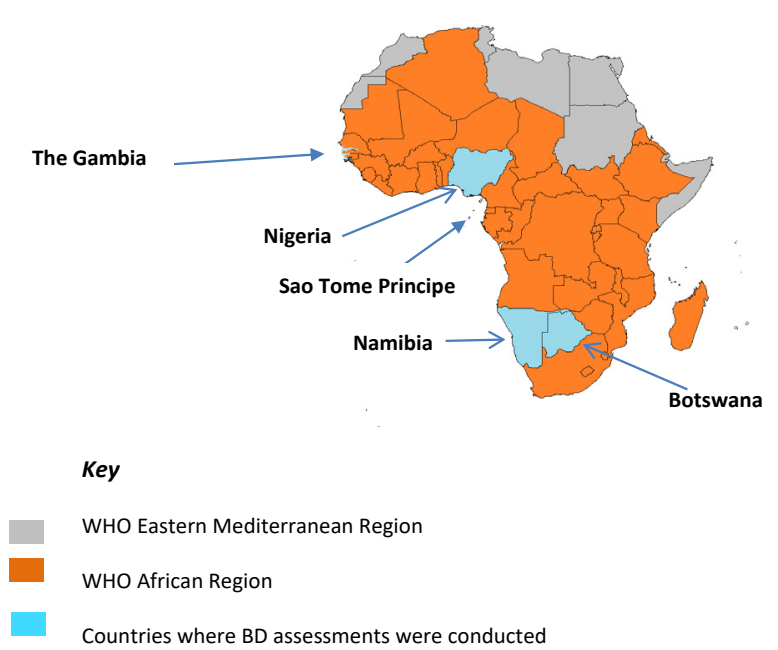

Figure 1: Map showing the 5 countries where BD assessments were conducted 
Attempts were made to include at least one high and one low performing province. Provinces with $<10 \%$ coverage were excluded a priori. In each province, convenience sampling was done to identify a sample of 6 to 8 health facilities that provided delivery services. The main national referral hospitals in each country were selected. Private facilities were deliberately selected in cities such as Lagos with a heavy private sector presence.

At national level, health policies and programme documents including previous EPI reviews and recent Gavi progress reports were reviewed to obtain basic programmatic data. A review of $\mathrm{WHO}$ and the United Nations Children's Fund (UNICEF) immunization coverage estimates which are based on data reported by countries through the Joint Reporting Form (JRF) was conducted. Interviews were conducted with Ministry of Health (MoH) officials regarding national HepB-BD and Maternal and Child Health (MCH) policies, training, supervision and vaccine management.

Evaluators drawn from $\mathrm{MoH}$ and $\mathrm{WHO}$ were trained to collect data using common assessment questionnaires which were developed during a February 2015 HepBBD consultative workshop which was held at the WHO
Regional Office for Africa (AFRO) in Brazzaville, Republic of Congo. The questionnaires contained structured and unstructured questions surrounding HepB-BD implementation. At each visited health facility, EPI and maternity supervisors were interviewed about HepB-BD vaccination policy, practices, knowledge and barriers, including those related to home births. They were questioned about any previous training in basic emergency obstetric and neonatal care (BEmONC), and whether the trainings endorsed the provision of timely HepB-BD. Evaluators observed vaccine handling and storage at each health facility. For each facility, data was collected on the number of births that had taken place in the year preceding the assessment, and the number of infants who had received a dose of HepB-BD. Finally, a record-based coverage assessment was conducted for each facility to calculate two estimates: (1) total HepB$\mathrm{BD}$ (doses administered any time after birth), and timely HepB-BD (doses given on the day of birth or the day after). A random sample of recent births was identified from the maternity register and later searched for in the immunization registry. The date of the vaccine dose was compared against the date of birth to determine the

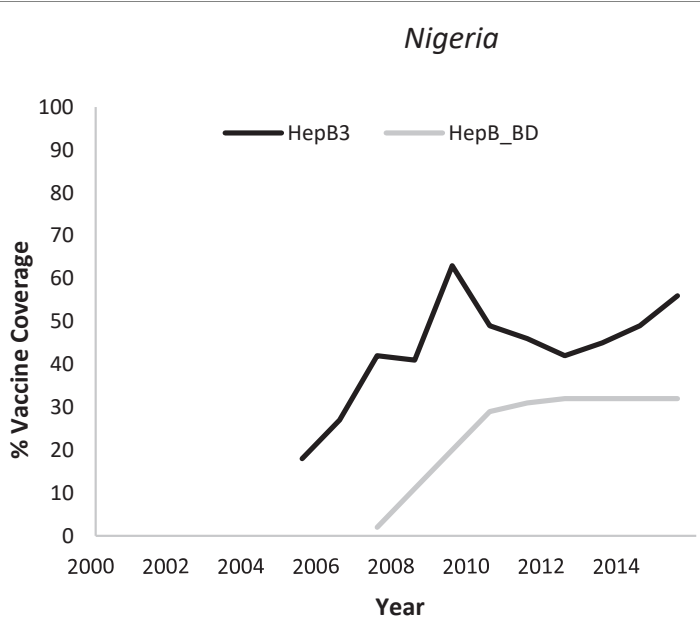

Namibia

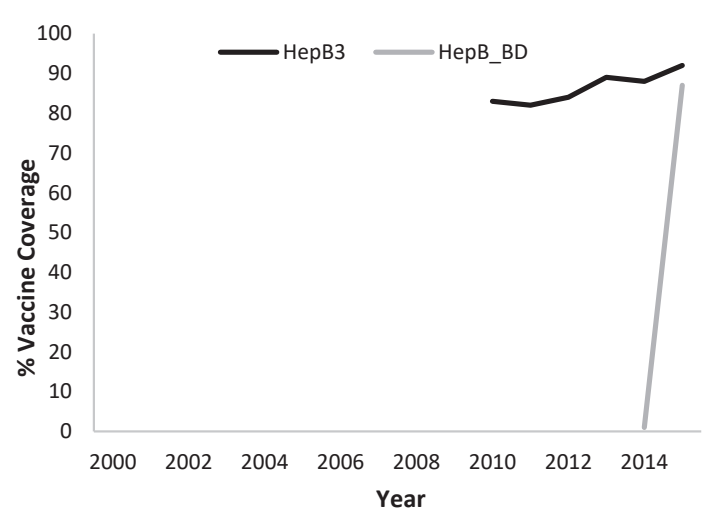

Gambia
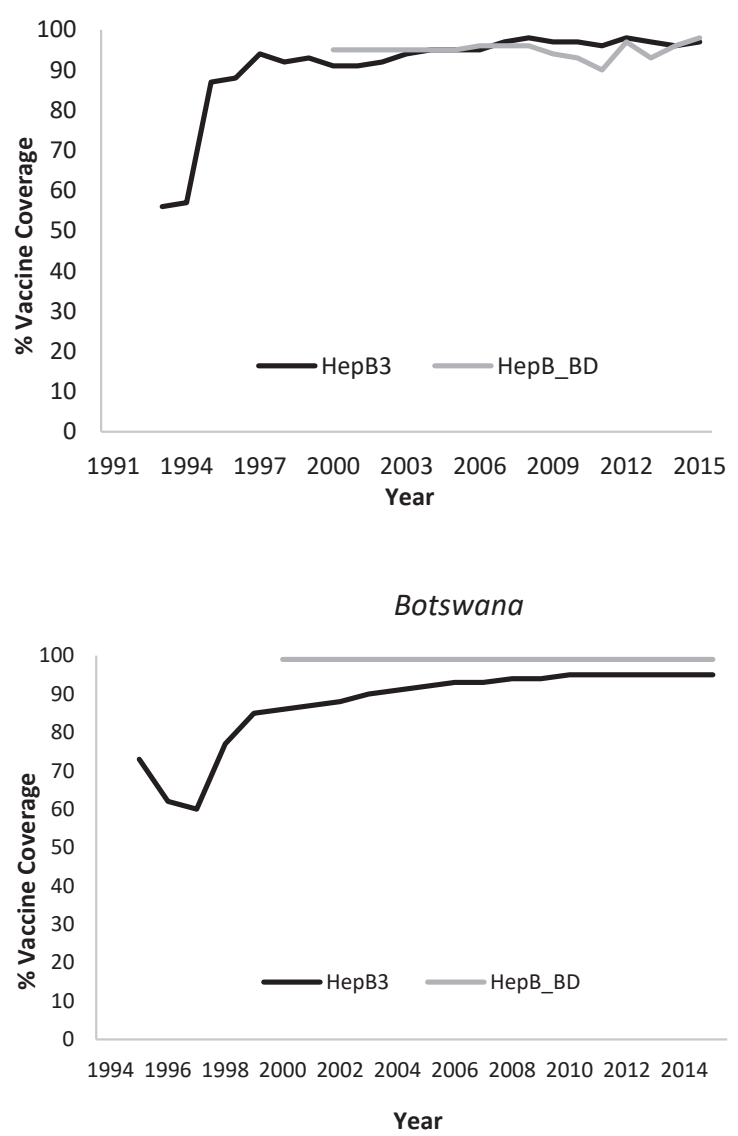

Figure 2: Childhood Hepatitis B vaccination coverage in the countries with birth dose assessments 
timing of HepB-BD administration. A modified protocol was employed in Namibia as the HepB-BD assessment was embedded in a larger comprehensive EPI and surveillance review and did not include a record-based coverage assessment component.

Data were entered, cleaned and analyzed using a Microsoft Excel database ${ }^{26}$. Descriptive statistics like frequencies and proportions were used to summarize the data. The assessments were funded by WHO and were exempted from ethical approval for human subjects from institutional review boards.

\section{Results}

A total of 78 health facilities were visited during the assessments: STP 5 (6\%), Nigeria 23 (29\%), Gambia 9 (12\%), Botswana 16 (21\%), and Namibia 25 (32\%) (Figure 1). The health facilities were a combination of public hospitals and health centers and private health facilities. All 78 facilities provided hepatitis B vaccination services (Table 1). The total number of deliveries per year in the selected health facilities ranged from 167 (range: 19-6,000) in Botswana to 1719 (range: 500-6,000) in the Gambia. All health facilities in STP were keeping mothers in the post-natal ward for at least 24 hours after delivery, as compared to facilities in Nigeria (43\%), the Gambia (33\%), and (13\%). (Figure 2)

A total of 1,196 records were evaluated in the three countries where capture-recapture sampling was conducted: Botswana (400), Nigeria (571), and the Gambia (225). HepB-BD coverage calculations were not possible in STP because maternal HBsAg status was not documented in delivery registers. High total HepB-BD estimates were attained in the Gambia 84\% (range: 60-100\%), though

Table 1: Facility characteristics and vaccine knowledge, practices, and management for the countries with Hepatitis B birth dose assessments ${ }^{1}$

\begin{tabular}{|c|c|c|c|c|}
\hline & STP $(n=5)$ & Nigeria $(n=23)$ & Gambia $(n=9)$ & Botswana ( $n=16)$ \\
\hline \multicolumn{5}{|l|}{ Background characteristics } \\
\hline Total number of deliveries (median [range]) & $336(31-4,383)$ & $278(25-1,667)$ & $1719(500-6,000)$ & $167(19-6,000)$ \\
\hline $100 \%$ of mothers stay $>=24 \mathrm{~h}$ post delivery & $5(100 \%)$ & $10(43 \%)$ & $3(33 \%)$ & $2(13 \%)$ \\
\hline \multicolumn{5}{|l|}{ Staff Knowledge } \\
\hline Received training on $Н$ ep $B-B D$ & $4(80 \%)$ & $14(61 \%)$ & $6(56 \%)$ & $2(13 \%)$ \\
\hline Know that a mother can transmit HBV to her baby & $5(100 \%)$ & $23(100 \%)$ & $8(92 \%)$ & $12(75 \%)$ \\
\hline $\begin{array}{l}\text { Know that recommended HepB-BD administration is }<24 h \text { of } \\
\text { birth }\end{array}$ & $5(100 \%)$ & $23(100 \%)$ & $7(71 \%)$ & $14(88 \%)$ \\
\hline \multicolumn{5}{|l|}{ Practices } \\
\hline Vaccinate $A L L$ newborns with HepB-BD & $0(0 \%)$ & $23(100 \%)$ & $9(100 \%)$ & $16(100 \%)$ \\
\hline Follow standard written protocols for HepB-BD administration & $0(0 \%)$ & $6(26 \%)$ & $0(100 \%)$ & $0(100 \%)$ \\
\hline Provide written documentation of HepB-BD to mother & $5(100 \%)$ & $22(96 \%)$ & $9(100 \%)$ & $16(100 \%)$ \\
\hline Vaccinate in the delivery room & $5(100 \%)$ & $6(26 \%)$ & $0(100 \%)$ & $1(6 \%)$ \\
\hline Administer to very low weight babies $(<2 \mathrm{~kg})$ & $4(80 \%)$ & $9(39 \%)$ & $2(22 \%)$ & $0(0 \%)$ \\
\hline Administer to premature babies & $3(60 \%)$ & $6(26 \%)$ & $1(11 \%)$ & $1(6 \%)$ \\
\hline Administer to ill but stable babies & $1(20 \%)$ & $7(30 \%)$ & $2(22 \%)$ & $1(6 \%)$ \\
\hline Provide HepB-BD outreach vaccination to home births & $0(0 \%)$ & $0(0 \%)$ & $0(0 \%)$ & $0(0 \%)$ \\
\hline Charge patient for HepB-BD administration & $0(0 \%)$ & $2(9 \%)$ & $0(0 \%)$ & $2(13 \%)$ \\
\hline Patients sometimes refuse HepB-BD & $0(0 \%)$ & $1(4 \%)$ & $0(0 \%)$ & $1(6 \%)$ \\
\hline Offer HepB-BD vaccination daily & $5(100 \%)$ & $0(0 \%)$ & $0(0 \%)$ & $16(100 \%)$ \\
\hline Require a physician order for HepB-BD & $0(0 \%)$ & $0(0 \%)$ & $0(0 \%)$ & $0(0 \%)$ \\
\hline \multicolumn{5}{|l|}{ Vaccine management } \\
\hline Stock out >2 weeks in 2014 & $0(0 \%)$ & $0(0 \%)$ & $0(0 \%)$ & $2(13 \%)$ \\
\hline Vaccine fridge is EPI-approved & $5(100 \%)$ & $12(52 \%)$ & $9(100 \%)$ & $16(100 \%)$ \\
\hline Observed VVM stage 3-4 in fridge & $0(0 \%)$ & $1(4 \%)$ & $0(0 \%)$ & $0(0 \%)$ \\
\hline Fridge monitored at least $2 x /$ day & $5(100 \%)$ & $6(26 \%)$ & $9(100 \%)$ & $16(100 \%)$ \\
\hline Vaccine obtained from MOH EPI & $5(100 \%)$ & $23(100 \%)$ & $9(100 \%)$ & $16(100 \%)$ \\
\hline Implement multi-dose vial policy & & $23(100 \%)$ & $9(100 \%)$ & $16(100 \%)$ \\
\hline
\end{tabular}

${ }^{1} \mathrm{~A}$ modified protocol was employed in Namibia as the HepB-BD assessment was embedded in a larger comprehensive EPI and surveillance review, and did not include comprehensive facility assessment component. 
the timely estimate was significantly lower $7 \%$ (range: 16-28\%). Only 9\% of infants who received HepB-BD in the Gambia were vaccinated within 24 hours of birth. The median days to receiving HepB-BD was 11 days (IQR: 6-16 days), and majority (42\%) were vaccinated between 8 to 14 days. Nigeria had low total $23 \%$ (range: $12-40 \%$ ), and timely HepB-BD coverage 13\% (range: 2-21\%). Botswana had high total HepB-BD coverage estimates (94\% [range: 80-100\%]), with the highest found in facilities in Kwaneng West province (98\%), and the lowest in Gaborone (89\%). However, Kwaneng West facilities had the lowest timely HepB-BD estimates (62\%). Private hospitals had the highest total coverage (99\%), and the National referral hospital followed closely at $80 \%$. Overall, timely HepB-BD coverage in Botswana was 74\% (range: $57-88 \%$ ).

\section{Overview of the National HepB-BD programmes}

All five countries are highly endemic for HBV (Table 2). However, none of the 5 countries had conducted nationally representative seroprevalence assessments. With the exception of STP, all are countries have policies for universal vaccination with monovalent HepB-BD given soon after birth, and 3 doses of pentavalent vaccine. STP introduced HepB-BD in 2002 and is using a selective screening and targeted vaccination approach. Women are routinely screened for HBsAg during antenatal care (ANC) visits, and only babies of HBV-seropositive (HBV+) mothers receive HepB-BD. HBV+ mothers were counselled on importance of delivering in hospitals to ensure their newborns receive HepB-BD and other postnatal care (PNC) services. In 2015, STP reported high national coverage for HepB3 (96\%) but low HepB-BD $(3 \%)^{14}$. This low rate is thought to be due to denominator issues which did not allow for appropriate statistical analysis. However, coverage among infants born to HBV+ women is high (92\%). Nigeria introduced HepB-BD in 2004 but the 2015 coverage estimates for both HepB-BD (32\%) and HepB3 (56\%) are low. The Gambia introduced HepB-BD in the 1990s and has consistently maintained high coverage rates for HepB-BD (98\%) and HepB3 (97\%). Botswana introduced HepB-BD in the 1990s and has maintained high $(90 \%)$ national coverage rates. Namibia introduced HepB-BD in 2014 and in 2015 attained a high post-introduction coverage of $87 \%$, and $92 \%$ coverage rate for HepB3.

HBV is a priority health issue in all five countries, and there is a strong political support to implement HepBBD. All countries had national policies for the timing of HepB-BD administration, ranging from within 24 hours in Nigeria to up to 2 weeks after birth in Namibia (Table 3). A change in the vaccination policy in Nigeria in February 2015 restricted HepB-BD administration to only within 24 hours of birth and EPI tools were revised accordingly. National EPI reporting and recording tools in all 5 countries had designated columns for HepB-BD, though some health facilities were still using outdated versions

Table 2: Hepatitis B birth dose coverage, institutional births, and antenatal care visits in the 5 African countries with birth dose assessments

\begin{tabular}{|c|c|c|c|c|c|c|}
\hline Country & $\begin{array}{l}\text { \% HBsAg prevalence } \\
\text { (min \%, max \%) }\end{array}$ & $\begin{array}{l}\text { Year HepB-BD } \\
\text { introduced }\end{array}$ & $\begin{array}{l}\text { Annual Births } \\
(1000 s)^{1}\end{array}$ & $\begin{array}{l}\text { Institutional } \\
\text { deliveries } \% 2\end{array}$ & $\begin{array}{l}\text { Births attended } \\
\text { by SBA } \%^{2}\end{array}$ & $\begin{array}{l}>1 \text { ANC } \\
\text { visit } \%^{2}\end{array}$ \\
\hline Botswana & $5.3,10.6(35-37)$ & Pre 2000 & 55 & 100 & 95 & 94 \\
\hline Gambia & $8.5,9.1(38-40)$ & 1990 & 83 & 63 & 57 & 86 \\
\hline Namibia & $7.8,13.6(41-43)$ & 2014 & 72 & 87 & 88 & 97 \\
\hline Nigeria & $6.7,17.2(44-46)$ & 2004 & 7,133 & 36 & 38 & 61 \\
\hline Sao Tome and Principe ${ }^{3}$ & $6.1,10(47)$ & 2002 & 6 & 91 & 93 & 98 \\
\hline
\end{tabular}

${ }^{1}$ Annual birth data is derived from the WHO Immunization Monitoring System (updated May 2016) http://apps.who.int/immunization_ monitoring/globalsummary.

${ }^{2}$ Data derived from UNICEF (updated February 2016) www.data.unicef.org

${ }^{3}$ Sao Tome and Principe does not offer the birth dose universally, but follow a selective policy where infants of mothers that test HBsAg are offered vaccine

Table 3: National level characteristics and policies for the 5 countries with birth dose assessments

\begin{tabular}{|c|c|c|c|c|c|}
\hline & STP & Nigeria & Gambia & Botswana & Namibia \\
\hline National plan focusing on prevention and control of viral hepatitis & No & No & No & No & No \\
\hline Designated govt. unit responsible for carrying out viral hepatitis activities & No & Yes & No & No & No \\
\hline National representative sero-survey data showing HBV burden & No & No & No & No & No \\
\hline National clinical guidelines for managing viral hepatitis & No & No & No & No & No \\
\hline National guidelines/policy related to HepB-BD vaccination & Yes & Yes & Yes & Yes & Yes \\
\hline Have an upper limit for timely HepB-BD vaccination ( $\leq 24 \mathrm{hrs}$.) & No & Yes & No & No & No \\
\hline EPI recording tools allow for capture of timely ( $\leq 24 \mathrm{hrs}$.) and total BD doses & No & No & Yes & No & No \\
\hline HepB-BD integrated in newborn care policy & No & No & No & No & No \\
\hline $\mathrm{MCH}$ data recording tools capture HepB-BD administration & No & No & No & Yes & No \\
\hline Outreach programs to vaccinate home births within 24 hours & No & No & No & No & No \\
\hline
\end{tabular}


which did not have these columns. Healthcare facilities routinely documented HepB-BD doses on EPI tally sheets and reporting forms, but none had modified maternity registers to include dedicated columns for HepB-BD. $\mathrm{MCH}$ and EPI policies were well integrated in STP which facilitated collaboration on HepB-BD implementation. The following gaps were observed: (1) none of the 5 countries had national viral hepatitis action plans, (2) none had conducted a nationally representative HBV serosurvey, (3) only Nigeria had designated the Human Immunodeficiency Virus (HIV) program to spearheaded the development of a draft national policy for viral hepatitis, though this was done without EPI involvement, (4) none had national clinical guidelines for managing viral hepatitis, (5) only Botswana recorded HepB-BD data in both EPI and delivery registers, (6) only the Gambia had adapted EPI recording/ reporting tools to capture both timely and total HepBBD doses (tools revised in 2015) (7) none had integrated HepB-BD in the BEmONC package, and (8) none had formal outreach programs to vaccinate babies born outside health facilities.

\section{Perspectives at the Service Delivery Level}

\section{Knowledge, practices and missed opportunities to timely HepB-BD coverage}

Staff frequently reported a lack of specific training for HepB-BD ranging from to $56 \%$ in the Gambia to $88 \%$ in Botswana. Despite this, knowledge regarding HBV was high among interviewed HCWs, and most healthcare workers (HCWs) in Botswana and STP knew that HepBBD should be administered within 24 hours for maximum effectiveness. However, most interviewed staff had suboptimal knowledge regarding the age-limits and correct contraindications for HepB-BD. Additionally, HCWs in Nigeria were observed to have higher awareness for Bacillus Calmette-Guérin (BCG) than HepB-BD. Vaccination was a highly acceptable intervention in all five countries and no community refusal was observed. Vaccination services were offered daily in health facilities in STP and Botswana, including weekends while mothers remained hospitalized in maternity facilities. Vaccination often took place in the EPI clinic and was offered free of charge in all public facilities. However, private facilities charged small service fees. Private facilities in Nigeria and Namibia received their vaccine supply from the $\mathrm{MoH}$ in exchange for submitting monthly reports.

None of the staff interviewed in the 5 countries required senior authorization to administer HepB-BD. The Gambia, STP, and Botswana reported high antenatal care (ANC) attendance. Moreover, STP (91\%) and Botswana (100\%) reported high rates of facility delivery In STP, pregnant women who presented for delivery in health facilities were assessed for their HBsAg status. Those with no proof of
ANC attendance, either ANC card or clinic records, were tested again in order to determine whether their infant was eligible for vaccination. False contraindications were commonly reported, including not vaccinating premature, very low birth-weight $(<2 \mathrm{~kg})$, or ill but stable infants. HCWs in STP delayed initiating breastfeeding until HepB$\mathrm{BD}$ administration for fear of transmission via breas tmilk.

\section{Administrative and documentation barriers to timely HepB-BD}

With the exception of a few facilities in Namibia (36\%) and Nigeria (26\%), none of the assessed health facilities had written protocols for HepB-BD. STP's policy of maternal screening and selective HepB-BD vaccination was unique among the evaluated countries and was identified as the principal barrier to achieving high HepB-BD coverage. With the exception of $2(13 \%)$ facilities in Botswana, none reported vaccine stock-outs of over one month in the year preceding the evaluation. All countries implemented the multi-dose vial policy. The quality of cold chain was good in all facilities in STP, the Gambia and Botswana. However, 52\% and $12 \%$ of facilities in Nigeria and Namibia respectively, lacked EPI-approved fridges. With the exception of one facility in Nigeria, no facilities had expired vaccines in the fridges. All facilities routinely documented HepB-BD on EPI tools, but only Botswana had revised their MCH tools to capture HepB-BD doses, while the Gambia had revised the EPI tools to capture both timely and total HepB-BD doses. District and national HepB-BD coverage calculations in all 5 countries did not specify the timing of HepB-BD vaccination. Few facilities analyzed immunization data and utilized findings for programmatic planning.

Major barriers to timely HepB-BD observed during the assessments included: (1) none of the 5 countries had outreach programs to vaccinate babies who were born outside health facilities, yet countries like Nigeria and the Gambia had high proportion of home births, (2) vaccinating babies at the time of discharge instead of at birth e.g., $50 \%$ of facilities in Botswana, (3) lack of awareness about appropriate age-limits among HCWs made them refuse to vaccinate eligible babies e.g., only $16 \%$ of HCWs in Namibia would administer HepB-BD if the baby was born at home and brought to health facilities even if within the 14 days, (4) cultural factors such as waiting until after the child's naming day (around 7 days) before bringing them for PNC in the Gambia and Nigeria, and (5) lack of vaccination services during weekends and public holidays.

\section{Discussion}

Findings from the HepB-BD assessments demonstrate the variability of birth dose implementation and the challenges countries face in immunizing babies with timely dose of HepB-BD. In 2015, the coverage for HepB-BD in the African region was only $10 \%$, compared to the Global 
estimate of $39 \%{ }^{14}$. Among the 11 countries implementing HepB-BD, coverage ranged from $19 \%$ in Angola to $99 \%$ in Algeria and Botswana. Facility estimates reported in the assessments correlated to the national reported estimates. Overall, visited facilities recorded lower timely HepB-BD estimates when compared to total rates. This could not be compared to national estimates because not all countries report timely HepB-BD coverage in the Joint Reporting Form (JRF), though revisions are underway.

\section{Barriers to increasing HepB-BD coverage in AFR - from assessments}

Reaching babies within 24 hours of birth was a difficult problem in all five countries. Barriers to the timely administration of HepB-BD included weaknesses in national policies and lack of written guidelines to standardize HepBBD implementation at health facilities. Evaluators did not find standard operating procedures (SOPs) at facilities to delineate when and where babies ought to be vaccinated, and which staff cadre was responsible for HepB-BD. As a result, many facilities were vaccinating babies at the point of hospital discharge instead of at birth. Vaccination services were often offered for half a day on weekdays and not on weekends or public holidays, and HepB-BD was not integrated into BEmONC packages in all countries.

Lack of training opportunities for HCWs was almost universally reported in all five countries. HepB-BD was often delayed or not given to infants because of inappropriate contraindication. Deficiency in knowledge about vaccine safety by HCWs has been linked to missed opportunities to vaccinate and low coverage rates ${ }^{27,28}$.

Previous assessments in the Gambia and the Philippines revealed the importance of increasing awareness and knowledge about HepB-BD vaccines among health workers to avoid false contraindication and missed opportunities to vaccinate ${ }^{20,21}$. The only contraindication to HepB-BD is anaphylactic reaction due to previous exposure. As such, all newborns are eligible for vaccination ${ }^{3}$. HCWs in STP withheld breastfeeding until HepB-BD vaccination because they falsely thought that breast milk could transmit HBV. Singling out HBV + mothers during ANC and labor could lead to stigmatization and make women fearful of delivering in hospitals.

\section{Opportunities to improve HepB-BD coverage - from assessments}

Governments in all five countries procured HepB$\mathrm{BD}$ and provided it free of charge in public and private facilities. Advocacy by stakeholders including physician/ nurses associations, the private sector, and hepatology associations in STP and Nigeria were integral in advocating for HepB-BD. Countries with a high proportion of institutional deliveries like Botswana (100\%) and STP
(91\%) achieved high coverage in part due to ease of accessing the babies ${ }^{29}$. High HepB-BD estimates in delivery facilities with on-site vaccination clinics, and having both services in the same facility made it convenient for mothers to access vaccination services thereby increasing coverage.

Botswana, the Gambia and STP which attained high coverage estimates, have high ANC attendance rates which provide opportunities for educating mothers on HepB-BD and importance of delivering in facilities. Most facilities in STP were retaining mothers in the post-natal wards for at least 24 hours after delivery, which presents an opportunity to vaccinate newborns. These assessments demonstrate the positive impact that offering daily vaccination services, including weekends and public holidays have on increasing coverage. Facilities that vaccinated babies in the delivery wards at time of birth reported higher coverage than those vaccinating at the point of discharge. With the heavy workload that HCWs face, it is important to provide written guidelines, refresher trainings and supportive supervision to emphasize HepB-BD vaccination. One private hospital in Nigeria that had high coverage developed a checklist form for staff to fill out prior to discharging mothers from the labor ward. Such tools remind HCWs to administer HepBBD and might improve HepB-BD coverage. Facilities in STP that stored HepB-BD in labor wards also had high coverage estimates. It was also observed that most facilities had high coverage rates of birth vaccines (BCG and first dose of polio vaccine [OPV]), which presents an opportunity to incorporate HepB-BD into essential newborn care package.

These assessments were conducted in a convenience sample of health facilities, which were located in purposively selected regions. Therefore, the findings might not be generalizable to the whole country or other countries in the region. Also, the facility coverage estimates reported in this document are not systematic so are not representative estimates of the true HepB-BD coverage in the five countries. However, they provide an additional data point with which to compare official national coverage estimates.

\section{Next steps/recommendations for introducing HepB- BD}

A large amount of work remains to be done in the region to achieve the control goal. All five countries had considerable challenges in achieving high timely HepBBD among both babies born in facilities and those born outside health facilities. While it is generally thought that vaccinating facility births is relatively straight forward and easy, these assessments show the missed opportunities and factors that contribute to low coverage among this cohort. They also demonstrate the enormous challenge countries will face in accessing babies born outside health facilities with timely HepB-BD. Countries seeking to introduce HepB- 
BD should offer universal vaccination to all newborns. Studies have found the targeted strategy to be ineffective ${ }^{30}$. If countries chose to implement targeted vaccination, they should develop systems to record maternal HBV status in delivery registers so babies can be tracked.

HCWs should be educated about the importance of timeliness of HepB-BD and provided with written guidelines and tools to augment their knowledge. Trainings should address knowledge gaps among HCWs and provide a consistent message: HepB-BD should be given ideally within 24 hours for maximum effectiveness, but if not possible, then it should still be given later. This difference should be distinguished in recording and reporting. Trainings should involve both public and private HCWs since both play an integral role in HepB-BD vaccination. Health facilities should develop SOPs to administer HepB$\mathrm{BD}$ as soon after birth as possible, rather than on discharge, and offer vaccination services every day including weekends and public holidays. The JRF global standard indicator for HepB-BD is defined as doses given $\leq 24$ hours after birth. However, JRF forms from the assessed countries did not have allowances to capture timing of HepB-BD. Countries should revise their recording and reporting tools to document timely birth doses, and any dose that is given before the first pentavalent vaccine. Reporting the two categories will help countries to account for all given doses. National delivery registers should also be modified to include dedicated columns for HepB with allocations for recording the date of administration.

Coordination between EPI and MCH is necessary to develop innovative strategies for reaching home births with timely HepB-BD. Babies who are born at home traditionally have lower vaccination coverage because they have less access to healthcare services. However, some births taking place outside health facilities might be attended to by skilled birth attendants (SBA), presenting an opportunity to vaccinate these babies ${ }^{29}$. HCWs also have a chance to counsel mothers on HepB-BD and importance of delivering in health facilities during ANC. This is a feasible strategy since it has been shown that $>90 \%$ of pregnant women in 27 African countries attended at least one ANC visit, and $80-90 \%$ of pregnant women in an additional 11 countries had at least one ANC visit ${ }^{22}$. Assurance of timely HepB-BD can be an added impetus for women who have delivered at home to seek Post Natal Care. Many African countries have nomadic and semi-nomadic populations with a high proportion of home births that are not attended by SBAs. It is also difficult to estimate vaccine coverage in this population because of unclear denominators. Using community health workers to track pregnancies to deliver targeted messages and offer home-based PNC may improve timely HepB-BD and is a feasible approach.

Several strategies have been employed successfully in countries in the Western Pacific Region to increase HepBBD administration to home births and can be implemented in Africa ${ }^{22,31}$. Use of vaccine out-of-cold chain (OCC) is a policy that might improve timely HepB-BD coverage in countries with low cold chain capacity and can support outreach vaccination services $^{31}$. In October 2016, the WHO Strategic Advisory Group of Experts (SAGE) encouraged the re-labelling of monovalent HepB for OCC use and that countries should follow the Immunization Practices Advisory Committee recommendations for $\mathrm{OCC}^{23}$. Several countries indicated their interest in introducing HepB-BD in national EPI in the next few years during a Regional WHO consultation held in November 2016 ${ }^{15}$. However, there is a need to conduct representative serological surveys to determine the true burden of chronic HBV and assess the impact of HepB vaccination. Findings from the sero-surveys can then be used evidence for national immunization technical advisory groups to approve HepB$\mathrm{BD}$ introduction, thereby accelerating steps to achieve the regional goal. WHO has developed guidelines to support countries ${ }^{12,18,32}$. The renewed momentum to control viral hepatitis in Region is an opportunity for countries to invest in HepB-BD introduction which has been shown to be a cost-effective intervention ${ }^{33,34}$.

\section{Conflict of Interest}

\section{None}

\section{References}

1. Perz JF, Armstrong GL, Farrington La, et al. The contributions of hepatitis $B$ virus and hepatitis $C$ virus infections to cirrhosis and primary liver cancer worldwide. J Hepatol. 2006; 45(4): 529-38.

2. WHO World Health Organization. Hepatitis B, Fact sheet $n^{\circ} 204$ [Internet]. Hepatitis B. 2013. p. 3. Available from: http://www.who. int/mediacentre/factsheets/fs204/en/

3. World Health Organization. Hepatitis B vaccines: WHO position paper. Wkly Epidemiol Rec [Internet]. 2009;84(40):405-20. Available from: http://www.ncbi.nlm.nih.gov/pubmed/19896455\%5Cnhttp:// www.who.int/wer

4. Shepard CW, Simard EP, Finelli L, et al. Hepatitis B virus infection: Epidemiology and vaccination. Epidemiologic Reviews. 2006; Vol 28: p. 112-25.

5. Wang H, Liddell CA, Coates MM, et al. Global, regional, and national levels of neonatal, infant, and under-5 mortality during 1990-2013: A systematic analysis for the Global Burden of Disease Study 2013. The Lancet. 2014; Vol 384: p. 957-79.

6. Goldstein ST, Zhou F, Hadler SC, et al. A mathematical model to estimate global hepatitis B disease burden and vaccination impact. Int J Epidemiol. 2005; 34(6): 1329-39.

7. Pierre Van Damme, John Ward, Daniel Shouval, et al. Vaccines. In: Vaccines. Elsevier Ltd. 2013; p. 205-34.

8. worldometers [Internet]. Africa Population. Available from: http:// www.worldometers.info/world-population/africa-population/

9. Schweitzer A, Horn J, Mikolajczyk RT, et al. Estimations of worldwide prevalence of chronic hepatitis B virus infection: a systematic review of data published between 1965 and 2013. Lancet (London, England) [Internet]. 2015; 6736(15): 1-10. Available from: http://www.ncbi. nlm.nih.gov/pubmed/26231459 
10. Lemoine M, Eholié S, Lacombe K. Reducing the neglected burden of viral hepatitis in Africa: Strategies for a global approach. J Hepatol [Internet]. 2015 Feb [cited 2017 Jan 27]; 62(2): 469-76. Available from: http://www.ncbi.nlm.nih.gov/pubmed/25457207

11. WHO Regional Office for Africa. African Regional Statistical Overview [Internet]. WHO. Available from: http://www.aho.afro.who.int/ profiles_information/images/2/24/AFRO-Statistical_Overview.pdf

12. WHO World Health Organization. Practices to improve coverage of the hepatitis B birth dose vaccine [Internet]. 2013. Available from: http:// apps.who.int/iris/bitstream/10665/78616/1/WHO_IVB_12.11_eng. pdf?ua $=1$

13. John Edmunds, Edward Jones, Andrea Apolloni, et al. Model estimates of the impact of HBV vaccination policies: Predicted current under 5 carriage. In 2016. Available from: http://www.who.int/ immunization/sage/meetings/2016/october/Session9-Estimatingthe-impact-of-HBV-vaccination-policies.pdf

14. WHO World Health Organization. WUENIC Data [Internet] Available from: http://apps.who.int/immunization_monitoring/ globalsummary/timeseries/tswucoveragebcg.html

15. WHO World Health Organization. World Health Organisation. Review of the barriers to implement the birth dose of hepatitis B. SAGE meeting of October 2016 2016. Available from: http://www.who. int/immunization/sage/meetings/2016/october/7_Review_of_the_ barriers_to_implement_the_birth_dose_of_hepb.pdf?ua=1.

16. WHO Regional Office for Africa. Regional Committee for Africa, Sixtyfourth session. In. Available from: http://www.afro.who.int/en/sixtyfourth-session.html

17. IASC. Mental Health and Psychosocial Support in Ebola Virus Disease Outbreaks: A Guide for Public Health Programme Planners [Internet] IASC Reference Group on Mental Health and Psychosocial Support in Emergency Settings. 2015. Available from: http://www.who.int/ mental_health/emergencies/ebola_guide_for_planners.pdf

18. WHO. A Guide for Introducing and Strengthening Hepatitis B Birth Dose Vaccination. Available from: http://apps.who.int/iris/ bitstream/10665/208278/1/9789241509831_eng.pdf

19. Howell J, Lemoine M, Thursz M. Prevention of materno-foetal transmission of hepatitis B in sub-Saharan Africa: the evidence, current practice and future challenges. J Viral Hepat [Internet]. 2014 Jun [cited 2017 Jan 27]; 21(6): 381-96. Available from: http://www. ncbi.nlm.nih.gov/pubmed/24827901

20. Miyahara R, Jasseh M, Gomez P, et al. Barriers to timely administration of birth dose vaccines in The Gambia, West Africa. Vaccine. 2016; 34(29): 3335-41.

21. Patel MK, Capeding RZ, Ducusin JU, et al. Findings from a hepatitis B birth dose assessment in health facilities in the Philippines: opportunities to engage the private sector. Vaccine [Internet]. 2014 Sep 3 [cited 2017 Feb 22]; 32(39): 5140-4. Available from: http:// www.ncbi.nlm.nih.gov/pubmed/24361121

22. Breakwell, Lucy, Tevi-Benissan, et al. Achieving Hepatitis B Control in the African Region: a case for hepatitis B vaccine birth dose introduction. Pan African J. 2016.

23. WHO World Health Organization. Summary of the October 2016 meeting of the Strategic Advisory Group of Experts on immunization (SAGE) [Internet]. 2016. Available from: http://www.who.int/ immunization/sage/meetings/2016/october/SAGE_October_2016_ Meeting_Web_summary.pdf?ua $=1$

24. Oku A, Oyo-Ita A, Glenton C, et al. Factors affecting the implementation of childhood vaccination communication strategies in Nigeria: a qualitative study. BMC Public Health [Internet]. 2017 Dec 15 [cited 2017 Feb 24]; 17(1): 200. Available from: http://www.ncbi.nlm.nih. gov/pubmed/28202001

25. Favin M, Steinglass R, Fields R, et al. Why children are not vaccinated: a review of the grey literature. Int Health. 2012; 4(4): 229-38.
26. Katz A. Microsoft Excel 2010. Style (DeKalb, IL). 2010; 21-39.

27. Wilson RJ, Paterson P, Jarrett C, et al. Understanding factors influencing vaccination acceptance during pregnancy globally: A literature review. Vaccine [Internet]. 2015 Nov 25 [cited 2017 Feb 22]; 33(47): 6420-9. Available from: http://www.ncbi.nlm.nih.gov/ pubmed/26320417

28. Suresh PS, Thejaswini V, Rajan T. Factors associated with 2009 pandemic influenza A (H1N1) vaccination acceptance among university students from India during the post-pandemic phase. BMC Infect Dis [Internet]. 2011 Jul 29 [cited 2017 Feb 22]; 11(1): 205. Available from: http://bmcinfectdis.biomedcentral.com/ articles/10.1186/1471-2334-11-205

29. UNICEF. UNICEF [Internet]. Available from: https://data.unicef.org/

30. Børresen ML, Koch A, Biggar RJ, et al. Effectiveness of the targeted hepatitis B vaccination program in Greenland. Am J Public Health [Internet]. 2012 Feb [cited 2017 Jan 22]; 102(2): 277-84. Available from: http://ajph.aphapublications.org/doi/10.2105/ AJPH.2011.300239

31. Xeuatvongsa A, Datta SS, Moturi E, et al. Improving hepatitis B birth dose in rural Lao People's Democratic Republic through the use of mobile phones to facilitate communication. Vaccine. 2016; 34(47): 5777-84.

32. WHO World Health Organization. Documenting the Impact of Hepatitis B Immunization: best practices for conducting a serosurvey. 2011.

33. Hall AJ, Roberston RL, Crivelli PE, et al. Cost-effectiveness of hepatitis B vaccine in The Gambia. Trans R Soc Trop Med Hyg [Internet]. 1993 May [cited 2017 Feb 24]; 87(3): 333-6. Available from: https:// academic.oup.com/trstmh/article-lookup/doi/10.1016/00359203(93)90154-I

34. Griffiths UK, Hutton G, Das Dores Pascoal E. The cost-effectiveness of introducing hepatitis $B$ vaccine into infant immunization services in Mozambique. Health Policy Plan [Internet]. 2005 Jan 1 [cited 2017 Feb 24]; 20(1): 50-9. Available from: http://www.ncbi.nlm.nih.gov/ pubmed/15689430

35. Wester CW, Bussmann H, Moyo S, et al. Serological evidence of HIVassociated infection among HIV-1-infected adults in Botswana. Clin Infect Dis. 2006; 43(12): 1612-5.

36. Patel P, Davis S, Tolle M, et al. Prevalence of Hepatitis B and Hepatitis C Coinfections in an Adult HIV Centre Population in Gaborone, Botswana. Am J Trop Med Hyg [Internet]. 2011 Aug 1 [cited 2017 Jan 22]; 85(2): 390-4. Available from: http://www.ajtmh.org/cgi/ doi/10.4269/ajtmh.2011.10-0510

37. Machiya T, Burnett RJ, Fernandes L, et al. Hepatitis B vaccination of healthcare workers at the Princess Marina Hospital, Botswana. Int Health [Internet]. 2015 Jul [cited 2017 Jan 22]; 7(4): 256-61. Available from: http://inthealth.oxfordjournals.org/lookup/doi/10.1093/ inthealth/ihu084

38. Mboto CI, Davies-Russell A, Fielder M, et al. Hepatitis C antibodies in asymptomatic first-time blood donors in The Gambia: prevalence and risk factors. Br J Biomed Sci [Internet]. 2005 [cited 2017 Jan 22]; 62(2): 89-91. Available from: http://www.ncbi.nlm.nih.gov/ pubmed/15997884

39. Mele A, Stroffolini T, Zanetti AR. Hepatitis B immunization and hepatocellular carcinoma: The Gambia Hepatitis Intervention Study. In: Journal of Medical Virology. 2002; p. 444-6.

40. Gambia T, Study H. The Gambia Hepatitis Intervention Study. The Gambia Hepatitis Study Group. Cancer Res. 1987; 47: 5782-7.

41. Vardas E, Sitas F, Seidel K, et al. Prevalence of hepatitis C virus antibodies and genotypes in asymptomatic, first-time blood donors in Namibia. Bull World Health Organ [Internet]. 1999 [cited 2017 Jan 22]; 77(12): 965-72. Available from: http://www.ncbi.nlm.nih.gov/ pubmed/10680243 
42. Joubert JJ, Prozesky OW, Lourens JG, et al. Prevalence of hepatitis virus and some arbovirus infections in Kavango, northern SWA/Namibia. S Afr Med J. 1985; 67(13): 500-2.

43. Steele AD, Bos P, Joubert JJ, et al. Serologic markers for hepatitis B virus and hepatitis A virus in Bushmen in West Caprivi, Namibia. East Afr Med J. 1995; 72(1): 30-2.

44. Fasola FA, Odaibo GN, Aken'Ova YA, et al. Hepatitis B and C viral markers in patients with sickle cell disease in Ibadan, Nigeria. Afr J Med Med Sci. 2003; 32(3): 293-5.
45. Uneke CJ, Ogbu O, Inyama PU, et al. Prevalence of hepatitis-B surface antigen among blood donors and human immunodeficiency virusinfected patients in Jos, Nigeria. Mem Inst Oswaldo Cruz. 2005; 100(1): 13-6.

46. Ikefuna A, Ilechukwu C, Emechebe G, et al. Hepatitis B virus infection in Nigeria - A review. Niger Med J. 2009; 50: 18-22.

47. Estatística IN de, Saúde M da, São Tomé ST e P. Inquérito Demográfico e Sanitário. 2009. 